\title{
Fuzzy Control Method for Synchronous Acquisition of High Resolution Image based on Machine Learning
}

\author{
Zichong Chen, Xianwen Luo* \\ School of Business College, Southwest University, Chongqing 402460, China \\ *Corresponding author: xianwenl@swu.edu.cn
}

Received: June 26, 2021. Revised: December 2, 2021. Accepted: January 11, 2022. Published: January 12, 2022.

\begin{abstract}
Aiming at the problem of low baud rate of traditional high-resolution image synchronous acquisition fuzzy control method, a high-resolution image synchronous acquisition fuzzy control method based on machine learning is designed. By detecting the fuzzy edge information of high-resolution image, the fuzzy membership function of synchronous acquisition quantity is proposed, and the gradient amplitude of synchronous acquisition quantity of high-resolution image is calculated. The unsupervised learning algorithm based on machine learning is used to cluster the fuzzy control data, so as to determine the fuzzy space of synchronous acquisition quantity of high-resolution image, and calculate the fuzzy feature similarity, the fuzzy control of synchronous acquisition quantity of high resolution image is realized. Experimental results show that the controlled wave rate in this paper solves the problem of low wave rate in $255.63 \mathrm{bps} / \mathrm{h}-271.33 \mathrm{bps} / \mathrm{h}$, and significantly improves the control accuracy.
\end{abstract}

Keywords-machine learning; high resolution image; synchronous acquisition volume; fuzzy control method.

\section{INTRODUCTION}

Image information is one of the most important information acquired by human beings. High resolution image acquisition is widely used in digital image processing, image recognition and other fields. High resolution image synchronous acquisition and processing plays an important role in modern multimedia technology. High resolution image synchronous acquisition is the core technology of digital camera, video phone, multimedia IP phone and teleconference products in daily life. The speed and quality of high resolution image acquisition directly affect the overall effect of products.

Yuan [1] designed an ultra-high-resolution photoelectric detection image preprocessing system to complete the post-processing and display task of the output images of the photoelectric detection device. The system can handle and display ultra-high-resolution images in real time, and can observe the images of different resolutions and the details of any part of the original image. In order to improve the acquisition and recognition ability of high-resolution images, $\mathrm{Xu}$ [2] proposed a super-resolution reconstruction method based on the OMP algorithm. Establish visual information image acquisition model, adopt visible image super resolution feature decomposition by spatial anchor neighborhood feature matching method, extract visible image edge profile features, estimate high-resolution image features fusion and optimization segmentation, establish visible image super resolution reconstruction combined with OMP algorithm. Mamdouh et al. [3] converted the (2 D) medical image data into a $3 \mathrm{D}$ model using Seg3D2 and ImageVis3D.Images were analyzed to create a liver. Image processing before and after the conversion phase was compared using the Python language program to ensure that the integrity of the image after the conversion process is the same as the original image of dicom without any distortion or change.

The sampling point of the above image acquisition method is difficult to correspond to the pixels of the camera one by one in the output sequence. After digitization, the video image quality loss is large, and the image resolution is also limited. Secondly, the hardware circuits of these methods are generally complex and of high cost, which are not conducive to the promotion and popularization.

In order to solve the shortcomings of the above research results, improve the wave rate of high-resolution images synchronously and make the collected images clearer, this paper designed a fuzzy control method for high-resolution image acquisition based on machine learning. The gradient amplitude of the synchronous capture amount of high-resolution images was calculated by detecting the fuzzy edge information of high-resolution images by the fuzzy membership function. Unsupervised learning-based on machine learning algorithm is used to cluster the fuzzy control data and calculate fuzzy feature similarity and realize fuzzy control over synchronous high-resolution image acquisition. Experimental results show that this method solves the problem of low wave rate of traditional methods and helps to improve image clarity.

Machine learning is a scientific research in which computer systems use algorithms and statistical models instead of explicit instructions in order to perform specific 
tasks effectively. It focuses on how computers simulate or realize human learning behaviors, so as to acquire new knowledge or skills, reorganize the existing knowledge structure, and constantly improve their own performance. It is the core of artificial intelligence and the fundamental way to make computer have intelligence. It is applied in all fields of artificial intelligence. It mainly uses induction, synthesis rather than deduction. Machine learning algorithm constructs a mathematical model based on sample data, which is called "training data", in order to predict or make decisions without explicit programming to perform tasks. Machine learning algorithms are used in a variety of applications, such as e-mail filtering and computer vision, where it is not feasible to develop algorithms for specific instructions to perform tasks. Machine learning can improve decision-making ability and correct mistakes. After machine learning, there is no need to issue fixed commands to keep following certain instructions to control the decision-making ability. The decision is made by machine learning without the intervention of fixed instructions. Machine learning algorithm can correct these errors after analyzing them, so as to improve efficiency and accuracy. Machine learning algorithm can manage and improve a large number of multi-dimensional data, and realize the function of accurate recognition, which helps to improve work efficiency. Therefore, this paper proposes a fuzzy control method based on machine learning for synchronous acquisition of high-resolution images.

\section{MACHINE LEARNING}

Machine learning is a scientific research that computer system does not use explicit instructions, but relies on Algorithms and statistical models used in pattern and reasoning in order to effectively perform specific tasks. It is seen as a subset of AI. Machine learning algorithm constructs a mathematical model based on sample data, called "training data", in order to predict or make decisions without explicit programming to perform tasks. Machine learning algorithms are used in a variety of applications, such as e-mail filtering and computer vision, where it is not feasible to develop algorithms for specific instructions to perform tasks. Machine learning is closely related to computational statistics, which focuses on computer prediction [4]. The research of algorithm optimization provides method, theory and application fields for machine learning. Data mining is a research field in machine learning, which focuses on exploratory data analysis to unsupervised learning. In the application of cross business problems, machine learning is also called predictive analysis. Machine learning tasks are divided into several categories. In supervised learning, the algorithm builds a mathematical model from a set of data including input and expected output. For example, if the task is used to determine whether an image contains an object, the training data of the supervised learning algorithm will include images with and without the object (input), and each image will have a label (output) to indicate whether it contains the object. In special cases, the input may be only partially available or limited to special feedback. The semi supervised learning algorithm develops a mathematical model based on incomplete training data, some of which have no labels.

Classification algorithm and regression algorithm are the types of supervised learning. When the output is limited to a limited set of values, the classification algorithm is used [5], [6]. For the classification algorithm of filtering e-mail, the input will be the e-mail received, and the output will be the name of the folder where the e-mail is archived. For spam recognition algorithm, the output will be the predicted value of "spam" or "non spam", which is represented by Boolean values true and false. Regression algorithms are named after their continuous output, which means they can have any value in the range. Examples of continuous values are the temperature, length, or price of an object.

In unsupervised learning, the algorithm builds a mathematical model from a set of data that contains only input but not output tags. Unsupervised learning algorithm is used to find the structure of data, such as the grouping or clustering of data points [7]. Unsupervised learning can discover patterns in data and group inputs by category, just as in feature learning. Dimensionality reduction is the process of reducing "features" or inputs in a set of data.

Active learning algorithm accesses the required output (training tag) according to a set of input with limited budget, and optimizes the input selection for obtaining training tag. When used interactively, these can be presented to human users for marking [8]. Reinforcement learning algorithm gives feedback in the form of positive or negative reinforcement in dynamic environment, and is used for automatic vehicles or learning to play games with human opponents. Other special algorithms in machine learning include topic modeling, in which the computer program is given a set of natural language documents and finds other documents covering similar topics [9]. Machine learning algorithm can be used to find the unobservable probability density function in the density estimation problem. Meta learning algorithm learns its own inductive bias based on previous experience [10]. In the development of robotics, robot learning algorithms generate their own learning experience sequences, also known as courses, which accumulate new skills through self-guided exploration and social interaction with human beings. These robots use active learning, maturity, movement cooperation and imitation.

\section{FUZZY CONTROL METHOD FOR SYNCHRONOUS}

\section{ACQUISITION OF HIGH RESOLUTION IMAGE BASED ON} MACHINE LEARNING

\section{A. Detection of fuzzy edge information of high} resolution image synchronous acquisition quantity

In the process of fuzzy control of synchronous acquisition amount of high-resolution image, the high-resolution image signal coding / decoding layer, image signal exchange layer and wireless connection network layer are used to preprocess the synchronous acquisition amount. According to the real-time TCP (transmission control protocol) protocol, high-resolution images can be 
acquired synchronously. In the process of detecting the fuzzy edge information of high-resolution images, the directionality of high-resolution images and the subtle difference between the edge information must be taken into account, Detect the fuzzy edge information of synchronous acquisition amount of high-resolution image [11]. The fuzzy edge information of the low frequency part is only the approximate component of the synchronous acquisition amount of the high resolution image; however, the high-frequency part of the fuzzy edge information has different degrees of sparsity [12]. The horizontal fuzzy edge information has column sparsity; the vertical fuzzy edge information has row sparsity; Diagonal fuzzy edge information has diagonal sparsity.

\section{B. The fuzzy membership function of synchronous} acquisition quantity of high resolution image is

\section{proposed}

On the basis of defining the fuzzy edge information of synchronous acquisition quantity of high-resolution image, the fuzzy membership function of synchronous acquisition quantity of high-resolution image is calculated based on machine learning [13], [14]. Assuming that the fuzzy membership function of synchronous acquisition quantity of high-resolution image is $E$, the calculation formula of $E$ is shown in Formula (1):

$$
E=\frac{1}{M N} \sum_{i}
$$

In Formula (1), $M$ refers to the sparse value of the blurred edge of the high frequency part of the synchronous acquisition amount of high-resolution image; $N$ refers to the approximate component of fuzzy edge in the low frequency part of high resolution image synchronous acquisition; $i$ refers to the number of adaptive transformations based on machine learning, which is a real number [15]. After obtaining the fuzzy membership function of synchronous acquisition quantity of high-resolution image, machine learning is used to transform the fuzzy membership function. Assuming that the transformed fuzzy membership function is $D$, the calculation formula of $D$ is shown in Formula (2):

$$
D=\frac{1}{M N} \sum_{i}\left(\frac{E}{C}\right)
$$

In Formula (2), $C$ refers to the edge vector of synchronous acquisition amount of high-resolution image. On the basis of Formula (2), the fuzzy membership function coding of synchronous acquisition quantity of high-resolution image is obtained, as shown in Table 1.

Table 1. Coding table of fuzzy membership function for synchronous acquisition quantity of high resolution image

\begin{tabular}{cccc}
\hline $\begin{array}{c}\text { Serial } \\
\text { number }\end{array}$ & $\begin{array}{c}\text { Fuzzy } \\
\text { membership } \\
\text { function } \\
\text { coding }\end{array}$ & $\begin{array}{c}\text { Value } \\
\text { range }\end{array}$ & $\begin{array}{c}\text { Absolute } \\
\text { error }\end{array}$ \\
\hline 1 & $E$ & 0 & 0.0850 \\
2 & $D$ & $-21,12$ & 0.1858 \\
\hline
\end{tabular}

\begin{tabular}{lccc}
\hline 3 & $E D$ & $-18,29$ & 0.2303 \\
4 & $D E$ & $-35,70$ & 0.1037 \\
5 & $E D D E$ & $-614,453$ & 0.1932 \\
\hline
\end{tabular}

According to Table 1, the key information of high-resolution image synchronous acquisition quantity is concentrated in the low-frequency information area, while the key information is less distributed in the high-frequency information area.

\section{Calculating the gradient amplitude of synchronous} acquisition of high resolution image

Taking the fuzzy membership function proposed above as an example, in order to improve the synchronous acquisition quantity of high-resolution image, the baud rate of fuzzy control is calculated with gradient amplitude as the key parameter [16]. Gradient amplitude calculation means that grad is the gradient matrix, and the mean value of gray gradient of all points is obtained. According to the gradient amplitude of the kernel, the matching gradient amplitude calculation set is generated:

Input: real time high resolution image edge detection task set T;

Output: the mapping of each high-resolution image acquisition volume and virtual host, and the mapping of virtual host and physical host;

(1) $\mathrm{RH} ; /^{*}$ Scroll through the task set $\mathrm{T}^{*} /$;

(2) While the new task $\mathrm{t} 1 \in \mathrm{T}$ reaches do;

(3) Delete the mapping relationship between $\mathrm{Rh}$ high-resolution image synchronous acquisition waiting task and virtual host, and update the ready time of virtual host;

(4) Task $\mathrm{t} 1$ is added to $\mathrm{RH}$;

(5) All tasks in RH are sorted by non-decreasing deadline;

(6) For task T1 belongs to RH do;

(7) End for;

(8) End whirl.

The mapping calculation process of high-resolution image acquisition body and virtual host is as follows: Assuming $N$ input records, the calculation time of gradient amplitude is $t_{r N}$; The rest operation time refers to $t_{c} ; k$ refers to the number of times based on the accounting method; $T_{b}$ refers to the communication cost based on accounting method; $T_{\text {map }}$ refers to the algorithm time based on kernel; The total time cost based on accounting method refers to $T_{\text {yar }}$, and the calculation formula of $T_{\text {yarm }}$ is as follows:

$$
T_{\text {yarm }}=T_{\text {map }}+T_{b}=\left(t_{r N}+t_{c}\right) k+T_{b}
$$

The high-resolution image edge pixels are divided into gradient amplitude $m(i, j)$ of points; if the gradient direction is set to $\theta(i, j)$, the following Formulas (4) and (5) can be obtained:

$$
\begin{aligned}
& m(i, j)=\sqrt{f_{(x)}(i, j)^{2}+f_{(y)}(i, j)^{2}} \\
& \theta(i, j)=\arctan \left[f_{(x)}(i, j) / f_{(y)}(i, j)\right]
\end{aligned}
$$

In the formula, $f_{(x)}(i, j)$ refers to the difference in the horizontal direction of the edge of the high-resolution 
image; $f_{(y)}(i, j)$ refers to the vertical difference of high resolution image edge. The direction of the gradient is the fastest change direction, when there are edges in the image, there must be a large gradient value, on the contrary, when the image of the smooth, gray value change is small, the corresponding gradient is smaller, considering the gray scale change in each pixel of the neighborhood, using the edge of the Robinson gradient operator, the gradient amplitude expression of the images is shown as follows:

$$
\begin{aligned}
G_{x} & =\xi f(x, y)-f(x-1, y) \\
G_{y} & =f(x, y)-\xi f(x, y-1)
\end{aligned}
$$

In the formula, $\xi$ express the Robinson gradient operator.

Thus, the gradient amplitude of image acquisition is obtained

\section{Fuzzy control data of synchronous acquisition} quantity of high resolution image based on Machine

\section{Learning Clustering}

According to the gradient amplitude of synchronous acquisition volume of high-resolution image calculated above, the fuzzy control data of synchronous acquisition volume of high-resolution image is clustered based on machine learning. In this process, the unsupervised learning algorithm based on machine learning divides the clustering of high-resolution images into two parts. First, high-resolution images are collected from network nodes through the map stage of unsupervised learning algorithm, and the high-resolution images are divided into several small data sets with data descriptive characteristics as indicators [17]. Then, in the reduce stage of unsupervised learning algorithm, unstructured data sets are represented. Supposing that the objective function of unstructured representation of high-resolution image data in reduce stage is $\omega$, as shown in Formula (8):

$$
\omega=t f(i, d)
$$

In Formula (8), $t$ refers to the attribution degree of high-resolution image data points; $f$ refers to the attraction between two high-resolution image sets; $i$ refers to the number of times the same high-resolution image appears in the synchronous acquisition volume; $d$ refers to the weight of high-dimensional features in high-resolution images.

Through Formula (8), the unstructured data set is obtained. Finally, the sparse representation technique in machine learning is used to represent high-resolution image features through low dimensional data in the same subspace. After unstructured data representation based on Reduce, this paper uses the method of calculating the subspace dimension to extract the features of the fuzzy control data of high-resolution image synchronous acquisition quantity, and realizes the clustering of the fuzzy control data of high-resolution image synchronous acquisition quantity based on machine learning. Assuming that the objective function of this process is $Y$, Formula (9) can be obtained:

$$
Y=\left[y_{1}, \ldots, y_{n}\right] \omega
$$

In Formula (9), it refers to the weight of high-dimensional feature space data in high-resolution image; it refers to the number of high-dimensional features in high-resolution image, which is a real number [18], [19]. Through Formula (9), the features of high-resolution image can be extracted, and the attractiveness of data points in high-resolution image can be updated in a distributed parallel way. By using the clustering function of machine learning, the fuzzy control data of synchronous acquisition volume of high-resolution image can be stored in a distributed way, providing basic data for its fuzzy control.

\section{E. Fuzzy space for determining synchronous}

\section{acquisition amount of high resolution image}

On the basis of machine learning clustering high-resolution image synchronous acquisition quantity fuzzy control data, assuming that the synchronous acquisition quantity of high-resolution image is $V$, the fuzzy space diagram of $V$ information feature is shown in Figure 1.

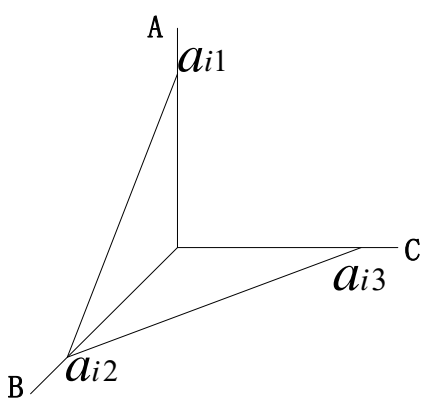

Figure 1. Fuzzy space diagram of synchronous acquisition quantity of high resolution image

In Figure 1, it can be seen that the fuzzy space of synchronous acquisition amount of high-resolution image is a three-dimensional space, in which $\mathrm{A}, \mathrm{B}$ and $\mathrm{C}$ are the three mapping lines of synchronous acquisition amount information features of high-resolution image in fuzzy space, which can show the specific attributes of synchronous acquisition amount information features of high-resolution image in fuzzy space [20]. However, $a_{i 1}$, $a_{i 2}$ and $a_{i 3}$ can show the potential relationship between the characteristics of high-resolution image synchronous acquisition amount and space. The more obvious the characteristics of high-resolution image synchronous acquisition amount, the larger the angle and the larger the space dimension; On the contrary, the less obvious the feature of synchronous acquisition amount of high-resolution image, the smaller the angle and the smaller the spatial dimension. By determining the fuzzy space of high-resolution image synchronous acquisition feature, the spatial attribute of high-resolution image synchronous acquisition feature can be judged, and the fuzzy feature similarity can be calculated as the limited range [21]. In this paper, Hamming distance is taken as the standard to calculate the similarity of fuzzy features of high-resolution images. Assuming that it is $\operatorname{Sim}(x, y)$, the Formula (10) can be obtained: 


$$
\operatorname{Sim}(x, y)=1-\frac{\sum_{i=1}^{n}\left(V \times \sum_{k}^{m} w_{i} \times|\mu(x)-\mu(y)|\right)}{\sum_{i=1}^{g}\left(V \times \sum_{k}^{m} w_{i} \times \operatorname{Max}(\mu(x), \mu(y))\right)}(10)
$$

In Formula (10), $m$ refers to the friction coefficient; $k$ is the feature weight coefficient of high resolution image synchronous acquisition; $g$ is the fuzzy feature weight coefficient; $\mu$ is the feature threshold of the overall shape of high resolution image; $x$ is the abscissa of high resolution image features in fuzzy space; $y$ refers to the ordinate of high-resolution image features in fuzzy space. According to the abscissa and ordinate of high-resolution image features in the fuzzy space, the similarity threshold of high-resolution image features can be directly determined. This process is the description of fuzzy features of high-resolution image synchronous acquisition volume [22], [23]. Based on Formula (10), the larger the fuzzy feature value of the basic features of high-resolution image synchronous acquisition quantity, the higher the similarity, which has nothing to do with the fuzzy distance attribute. The most important parameters that affect the fuzzy feature value include the color, texture, shape and other information of high-resolution image.

\section{F. Realizing the fuzzy control of synchronous} acquisition quantity of high resolution image

In the process of fuzzy control of synchronous acquisition amount of high-resolution image, finally, in order to avoid overload of control bearing processing amount, this paper calculates the incremental value of fuzzy control bearing processing amount of synchronous acquisition amount of high-resolution image to realize fuzzy control of synchronous acquisition amount of high-resolution image [24]. Supposing the objective function is $\Lambda P$, then there is Formula (11):

$$
\Lambda P=P_{T 0}-P_{T}
$$

In Formula (11), $P_{T 0}$ refers to the synchronous acquisition amount of high-resolution image and the length of fuzzy control character; $P_{T}$ is the average number of matches. Through Formula (11), the incremental value of synchronous acquisition quantity of high-resolution image is calculated, and on this basis, the fuzzy control equation of synchronous acquisition quantity of high-resolution image is obtained [25]. Suppose the objective function is $k$, then there is Formula (12):

$$
k= \begin{cases}m . t & \max (V *) \\ s . t 1 & \max E(A) \\ \text { s.t } 2 & \max E(U) \\ \text { s.t3 } & \square P(w)>\left\{\left[\left(1-\theta_{1}\right)\right] \theta_{2}-r\right\} / \phi\end{cases}
$$

In Formula (12), $E$ refers to the length of Labview window function of fuzzy control signal of high resolution image synchronous acquisition quantity; $A$ refers to the weight of fuzzy control; $U$ refers to the control signal acquisition frequency; $\theta_{1}$ is the probability that the control can reach the expectation; $\theta_{2}$ refers to the probability that the control fails to reach the expectation; $\phi$ refers to cost control. Through Formula (12), the synchronous acquisition amount of high-resolution image is comprehensively controlled. Through the above control formula, the fuzzy control of synchronous acquisition quantity of high-resolution image is realized.

\section{EXPERIMENT}

In this paper, based on machine learning design a high-resolution image synchronous acquisition fuzzy control method, in order to test the application effect of the method, the following experiment.

\section{A. Experimental preparation}

In this paper, the baud rate of high-resolution image synchronous acquisition fuzzy control is quantitatively evaluated by constructing experiments. In this experiment, four high-resolution images are selected as the experimental objects in the Ueruben high-resolution image library, which are: big tree; train; the house and the plane. The bias parameters of four high-resolution images are set as the initial values; the damping coefficient is set to 0.65 . First of all, this paper uses the design method of fuzzy control of high-resolution image synchronous acquisition, through MATALB test control baud rate, and record, set it as the experimental group; Then the method proposed by Yuan [1] is used to control the synchronous acquisition of high-resolution image, and the baud rate is also controlled by MATALB test, and recorded, which is set as the control group. Therefore, the main content of this experiment is to test the control baud rate of the two methods. The higher the baud rate is, the higher the control efficiency is. Through 10 contrast experiments, the experimental data are recorded according to the control baud rate.

\section{B. Experimental results and analysis}

Arranging the experimental data, as shown in Table 2.

Table 2. Control baud rate comparison table

\begin{tabular}{ccc}
\hline $\begin{array}{c}\text { Test times } \\
(\text { Times })\end{array}$ & $\begin{array}{c}\text { Experimental } \\
\text { group control } \\
\text { baud rate }(\mathrm{bps} / \mathrm{h})\end{array}$ & $\begin{array}{c}\text { Control group control } \\
\text { baud rate }(\mathrm{bps} / \mathrm{h})\end{array}$ \\
\hline$(1)$ & 255.63 & 131.54 \\
$(2)$ & 255.64 & 119.23 \\
$(3)$ & 268.97 & 121.61 \\
$(4)$ & 270.45 & 143.43 \\
$(5)$ & 259.34 & 123.75 \\
$(6)$ & 265.24 & 130.65 \\
$(7)$ & 267.81 & 128.36 \\
$(8)$ & 271.33 & 129.61 \\
$(9)$ & 269.87 & 141.34 \\
$(10)$ & 258.49 & 126.72 \\
\hline
\end{tabular}

It can be seen from Table 2 that the lowest baud rate of the fuzzy control group is $255.63 \mathrm{bps} / \mathrm{h}$, which is significantly higher than that of the control group in the same amount of high-resolution image synchronous acquisition, which has practical promotion value. The reason for the advantageous results is that the gradient 
amplitude of the synchronous capture of high resolution images is calculated by the fuzzy membership function. Unsupervised learning algorithm is used to cluster the fuzzy control data and determine the synchronous acquisition amount of high-resolution images, thus improving the accuracy of fuzzy control.

\section{CONCLUSIONS}

To make clearer the acquired images, this paper designed a machine-learning-based fuzzy control method for synchronous high-resolution image acquisition. The gradient of the image synchronous capture was calculated by detecting the fuzzy edge information of the high-resolution image by the fuzzy membership function. Unsupervised learning algorithm was used to cluster the fuzzy control data, calculate the fuzzy feature similarity, and control the synchronous acquisition of high-resolution images. The experimental results show that the wave rate improves compared with the traditional fuzzy control method, which improves the control accuracy and has a good application effect.

However, there are some shortcomings in this paper that need to further improve the control accuracy. Due to the complexity of the synchronous acquisition of fuzzy control by high-resolution images, there are many interference factors in the machine learning process, and there are a training error and a generalization error, when the training data is insufficient, leading to the interference in the clustering process. Future studies can combine other techniques, such as validation, fitting, to decrease in error, make cluster control more precise and play a greater role in practical applications.

\section{REFERENCES}

[1] H. Q. Yuan, "Design for ultra-high resolution image pre-process system for photoelectric detection", Journal of China Academy of Electronics and Information Technology, vol. 8, pp. 830-835, 2019.

[2] Q. Q. Xu, "Super-resolution Reconstruction method of visible Image based on OMP algorithm", Laser Journal, vol. 41 (11), pp. 5, 2020.

[3] R. Mamdouh, H. M. El-Bakry, A. Riad and N. El-Khamisy, "Converting 2D-medical image files DICOM? into 3D- Models, based on image processing, and analysing their results with Python programming", WSEAS Transactions on Computers, vol. 19, pp. 10-20, 2020.

[4] S. Sargsyan and A. Hovakimyan, "Neural network effectiveness evaluation while the intersection of images in the receptor field", WSEAS Transactions on Information Science and Applications, vol. 17, pp. 172-176, 2020.

[5] O. Alfarraj, "A machine learning-assisted data aggregation and offloading system for cloud-IOT communication". Peer-to-Peer Networking and Applications, vol. 14, pp. 2554-2564, 2020.

[6] M. Sadighi, B. H. Motamedvaziri and M. A. Ahmadi, "Assessing landslide susceptibility using machine learning models: A comparison between ANN, ANFIS, and ANFIS-ICA", Environmental Earth Sciences, vol. 79, no. 24, pp. 1-14, 2020.
[7] M. Mishra, A. S. Bhatia and D. Maity, "Predicting the compressive strength of unreinforced brick masonry using machine learning techniques validated on a case study of a museum through nondestructive testing". Journal of Civil Structural Health Monitoring, vol. 10, no. 3, pp. 389-403, 2020.

[8] M. Jacob, M. P. Mani and J. C. Ye, "Structured low-rank algorithms: theory, magnetic resonance applications, and links to machine learning". IEEE Signal Processing Magazine, vol. 37, no. 1, pp. 54-68, 2020.

[9] J. S. Chou, D. N. Truong and T. L. Le, "Interval forecasting of financial time series by accelerated particle swarm-optimized multi-output machine learning system", IEEE Access, vol. 8, no. 1, pp. 14798-14808, 2020.

[10] M. Atwya and G. Panoutsos, "Transient thermography for flaw detection in friction stir welding: A machine learning approach". IEEE Transactions on Industrial Informatics, vol. 16, no. 7, pp. 4423-4435, 2020.

[11] M. Al-Saud, A. M. Eltamaly, M. A. Mohamed and A. Kavousi-Fard, "An intelligent data-driven model to secure intravehicle communications based on machine learning”, IEEE Transactions on Industrial Electronics, vol. 67, no. 6, pp. 5112-5119, 2020.

[12] G. Macias-Bobadilla, J. D. Becerra-Ruiz, A. A. Estévez-Bén and J. Rodríguez-Reséndiz. "Fuzzy control-based system feed-back by OBD-ii data acquisition for complementary injection of hydrogen into internal combustion engines-sciencedirect", International Journal of Hydrogen Energy, vol. 45, no. 51, pp. 26604-26612, 2020.

[13] S. Ling, H. Q. Wang and L. X. Peter, “Adaptive fuzzy dynamic surface control of flexible-joint robot systems with input saturation", IEEE/CAA Journal of Automatica Sinica, vol. 6, no. 1, pp. 100-110, 2019.

[14] S. Du, M. Wu, L. F. Chen, K. Zhou and W. Pedrycz, "A fuzzy control strategy of burn-through point based on the feature extraction of time-series trend for iron ore sintering process", IEEE Transactions on Industrial Informatics, vol. 16, no. 4, pp. 2357-2368, 2020.

[15] J. J. Eckert, L. Silva, F. G. Dedini and F. C. Corrêa, "Electric vehicle powertrain and fuzzy control multi-objective optimization, considering dual hybrid energy storage systems", IEEE Transactions on Vehicular Technology, vol. 69, no. 4, pp. 3773-3782, 2020.

[16]M. AI, P. J. Wang and W. Ma, "Research and application of smart streetlamp based on fuzzy control method", Procedia Computer Science, vol. 183, no. 4, pp. 341-348, 2021.

[17]K. Odry, R. Fullér, I. J. Rudas and P. Odry, "Fuzzy control of self- balancing robots: A control laboratory project", Computer Applications in Engineering Education, vol. 28, no. 3, pp. 512-535, 2020.

[18] S. Xie, Y. Xie, F. Li, Z. Jiang and W. Gui, "Hybrid fuzzy control for the goethite process in zinc production plant combining type-1 and type-2 fuzzy logics", Neurocomputing, vol. 366, no. 13, pp. 170-177, 2019.

[19] X. Wang, J. H. Park, K. She, S. Zhong and L. Shi, "Stabilization of chaotic systems with t-s fuzzy model and nonuniform sampling: A switched fuzzy control 
approach", IEEE Transactions on Fuzzy Systems, vol. 27, no. 6, pp. 1263-1271, 2019.

[20]B. Alefy, H. A. Shayanfar, S. Soleymani and F. Faghihi, "Improvement in two adjacent microgrids frequency using the ac-to-ac converter based on sugeno fuzzy control scheme", International Journal of Fuzzy Systems, vol. 21, no. 3, pp. 782-792, 2019.

[21]Z. Wang, Y. Zhao, H. Du and K. Cao, "Stability analysis of T-S fuzzy control systems subject to actuator saturation", International Journal of Fuzzy Systems, vol. 21, no. 8, pp. 2625-2631, 2019.

[22] Y. Pan and G. H. Yang, "A novel event-based fuzzy control approach for continuous-time fuzzy systems", Neurocomputing, vol. 338, no. 21, pp. 55-62, 2019.

[23] V. I. Chizhikov and E. V. Kurnasov, "Tactile identification of a surface by fuzzy control", Russian Engineering Research, vol. 39, no. 6, 492-498, 2019.

[24] A. T. Nguyen, T. Taniguchi, L. Eciolaza, V. Campos and M. Sugeno, "Fuzzy control systems: past, present and future", IEEE Computational Intelligence Magazine, vol. 14, no. 1, pp. 56-68, 2019.

[25] M. Wang, Z. Wang, Y. Chen and W. G. Sheng, "Observer-based fuzzy output-feedback control for discrete-time strict-feedback nonlinear systems with stochastic noises", IEEE Transactions on Cybernetics, vol. 50, no. 8, pp. 3766-3777, 2020.

\section{Creative Commons Attribution License 4.0}

\section{(Attribution 4.0 International, CC BY 4.0)}

This article is published under the terms of the Creative Commons Attribution License 4.0

https://creativecommons.org/licenses/by/4.0/deed.en_US 